\title{
Medicago spp. as potential sources of bioactive isoflavones: Characterization according to phylogenetic and phenologic factors
}

\author{
João C.M. Barreira ${ }^{\text {a,b,*, }}$, Tatiana Visnevschi-Necrasov ${ }^{\mathrm{a}, \mathrm{c}}$, Eugénia Nunes ${ }^{\mathrm{c}}$, Sara C. Cunha ${ }^{\mathrm{a}}$, Graça Pereira ${ }^{\mathrm{d}}$, \\ M. Beatriz P.P. Oliveira ${ }^{a}$ \\ ${ }^{a}$ REQUIMTE, Departamento de Ciências Químicas, Faculdade de Farmácia, Universidade do Porto, Rua Jorge Viterbo Ferreira, $n^{\circ} 228,4050-313$ Porto, Portugal \\ ${ }^{\mathrm{b}}$ CIMO-ESA, Instituto Politécnico de Bragança, Campus de Santa Apolónia, Apartado 1172, 5301-855 Bragança, Portugal \\ ${ }^{\mathrm{C}}$ CIBIO-ICETA, Faculdade de Ciências, Universidade do Porto, R. Padre Armando Quintas 4485-661, Vairão, Portugal \\ ${ }^{\mathrm{d}}$ INRB/IP - INIA - Instituto Nacional de Recursos Biológicos, Caia E São Pedro Estrada Gil Vaz, 7350-228 Elvas, Portugal
}

\section{A R T I C L E I N F O}

\section{Article history:}

Received 20 December 2014

Received in revised form 20 April 2015

Available online 13 May 2015

\section{Keywords:}

Isoflavones

Medicago

Phylogeny

Phenology

MSPD extraction

HPLC-DAD

GLM

LDA

\begin{abstract}
A B S T R A C T
A high variety of plant species are often proposed as potential natural sources of specific bioactive components, with emphasis in phenolic compounds. However, the ability to produce a determined phytochemical might be variable, even among species with close phylogeny. Furthermore, the metabolic dynamics vary greatly according to phenologic factors. Herein, it was verified whether isoflavone production in Medicago spp. is more associated with phylogenetic or phenologic determinants, to define the optimal productive conditions. Isoflavone profiles were characterized in field-grown Medicago species in three phenologic stages. Isoflavones were extracted by matrix solid-phase dispersion method and analyzed using high-performance liquid chromatography coupled with a diode-array detector. The obtained data were evaluated by a generalized linear model (GLM) and linear discriminant analysis (LDA). Formononetin, genistein and irilone were the most abundant isoflavones, reaching values higher than those present in acknowledged plant sources like soy or red clover. Outputs from GLM and LDA indicate that the phylogenetic factors are the most defining criteria. This study promotes Medicago spp. as potential isoflavone sources, particularly because the effects of these compounds are highly dependent on their type and concentration, with potential application as foodstuff, feedstuff, or in the nutraceutical and pharmaceutical industry.
\end{abstract}

(c) 2015 Elsevier Ltd. All rights reserved.

\section{Introduction}

The genus Medicago is part of the botanical family of Leguminosae and includes about 56 different species mainly distributed in Mediterranean climatic conditions areas (Farag et al., 2007). Besides alfalfa (Medicago sativa), which is the main Medicago species grown throughout the world, inclusively as source of phytochemicals (Nunes et al., 2008; Silva et al., 2013), not much attention has been given to other Medicago species. Medicago truncatula, which is often chosen as a model for genomic studies of Fabaceae due to its small diploid genome ( $\left.\approx 5 \times 10^{8} \mathrm{bp}\right)$, self-fertilization, easy genetic transformation and prolific nature, is the sole exception (Farag et al., 2007; Huhman and Sumner, 2002; Schliemann et al., 2008; Stochmal et al., 2009). Medicago species

\footnotetext{
* Corresponding author at: CIMO-ESA, Instituto Politécnico de Bragança, Campus de Santa Apolónia, Apartado 1172, 5301-855 Bragança, Portugal. Tel.: +351 273303909; fax: +351 273325405 .

E-mail address: jbarreira@ipb.pt (J.C.M. Barreira).
}

are important sources of phytochemicals, including carotenoids, saponins or phytoestrogens (Yildiz, 2005), which are known to act as antimicrobial agents, phytoanticipins, phytoalexins, structural barriers, modulators of pathogenicity, plant defense genes activators, or fungitoxic agents (Von Baer et al., 2006). Isoflavones are synthesized, accumulated and constitutively expressed as phytoalexins in response to pathogen attacks (Dakora and Phillips, 1996), contributing to the global strategies of plant defense mechanisms (Aloui et al., 2012) and modulation of the interaction of Fabaceae species with nitrogen-fixing bacteria in rhizobium-legume symbiosis (Antunes et al., 2006). Isoflavones are also known as having a wide range of beneficial biological activities in the human body (Mortensen et al., 2009), but their overconsumption have been suggested as potentially causing adverse effects (Setchell and Cassidy, 1999). Hence, the intake of isoflavones has been limited by International Organisation (such as Food Safety Commission of Japanese Government or The Nutrient Data Laboratory of the Agricultural Research Service of the United States Department of Agriculture) to very restricted 
values (Sakamoto et al., 2015). Nevertheless, the intake amounts of isoflavones are usually evaluated on the basis of aglycone forms because the isoflavone glycosides are converted into aglycones by intestinal flora in vivo and in vitro (Sakamoto et al., 2015).

The three main classes of phytoestrogens are isoflavones, lignans and coumestans (Jacobs et al., 2009), but their biosynthesis varies greatly with environmental and genotypic factors (Hoeck et al., 2000). Soybean, for instance, present mainly isoflavone aglycones (daidzein, glycitein and genistein) and glycoside, acetylglycoside and malonylglycoside forms. In contrast to soybean, red clover contains biochanin A and formononetin (aglycones), and their glycosides and malonyl derivatives as the major components, while in Medicago species, the most abundant isoflavones are formononetin, irilone and genistein (Visnevschi-Necrasov et al., 2014a). The isoflavones profile also varied greatly with the phenological stage, varying along the plant maturity (D'Agostina et al., 2008). In lupin plants, the content of isoflavones was reported as negligible in seeds when compared to the amounts detected in leaves and roots (Bednarek et al., 2001). In a different study with M. truncatula, constitutive root isoflavonoids, like formononetin malonyl glycoside or medicarpin malonyl glycoside, accumulate increasingly with the development of mycorrhizal symbiosis (Aloui et al., 2012). Furthermore, isoflavones profile is highly affected by genotype $\times$ environment interactions (Morrison et al., 2010).

Herein, the profiles in free and conjugated isoflavones were compared among open-field grown Medicago spp. and the changes along vegetative cycle were monitored by evaluating three different phenologic stages: vegetative elongation, late bud and late flower. With this approach, it was intended to evaluate the effect of the plant species and the phenologic stage (as well as the interaction of both factors) in the potential yield of individual and total isoflavones.

\section{Results and discussion}

\subsection{Phylogenetic and phenologic influence on isoflavone profiles}

In this study eleven isoflavones were quantified, eluting in the following order: (1) puerarin (7,4'-dihydroxy-8-C-glucosylisoflavone), (2) daidzin (daidzein-7-O- $\beta$-D-glucoside), (3) genistin (genistein-7-O- $\beta$-D-glucoside), (4) daidzein (4',7-dihydroxyisoflavone), (5) glycitein (4',7-dihydroxy-6-methoxyisoflavone), (6) genistein (4',5,7-trihydroxyisoflavone), (7) pratensein (4'-methoxy-3',5,7-trihydroxyisoflavone), (8) formononetin (7-hydroxy-4'methoxyisoflavone), (9) irilone (9-hydroxy-7-(4-hydroxyphenyl)-[1, 3]dioxolo[4,5-g]chromen-8-one), (10) prunetin (4',5-dihydroxy-7methoxyisoflavone) and (11) biochanin A (5,7-dihydroxy-4'methoxyisoflavone). Besides the chromatographic data, the proposed identification was also supported by UV spectra as described previously (Rodrigues et al., 2014). Chromatographic parameters, namely limit of detection (LOD), limit of quantification (LOQ), linearity, recovery and repeatability were accepted as previously assessed (Visnevschi-Necrasov et al., 2014a).

The effects of plant species (PSp) and phenologic stage (PhS), as well as the interaction of both factors, were assessed simultaneously by evaluating changes in isoflavones composition of Medicago spp. grown in open-field conditions. Studying the combined effect of both factors (PSp and PhS), allows understanding the influence of each single one, without having biased results; i.e., it is possible to identify the PSp with highest potential to produce a determined set of isoflavones independently of the $\mathrm{PhS}$ (which could be useful for monoculture crops) and also defining the most suitable PhS independently of the PSp (which would be particularly valuable in polyculture crops). Table 1 shows the

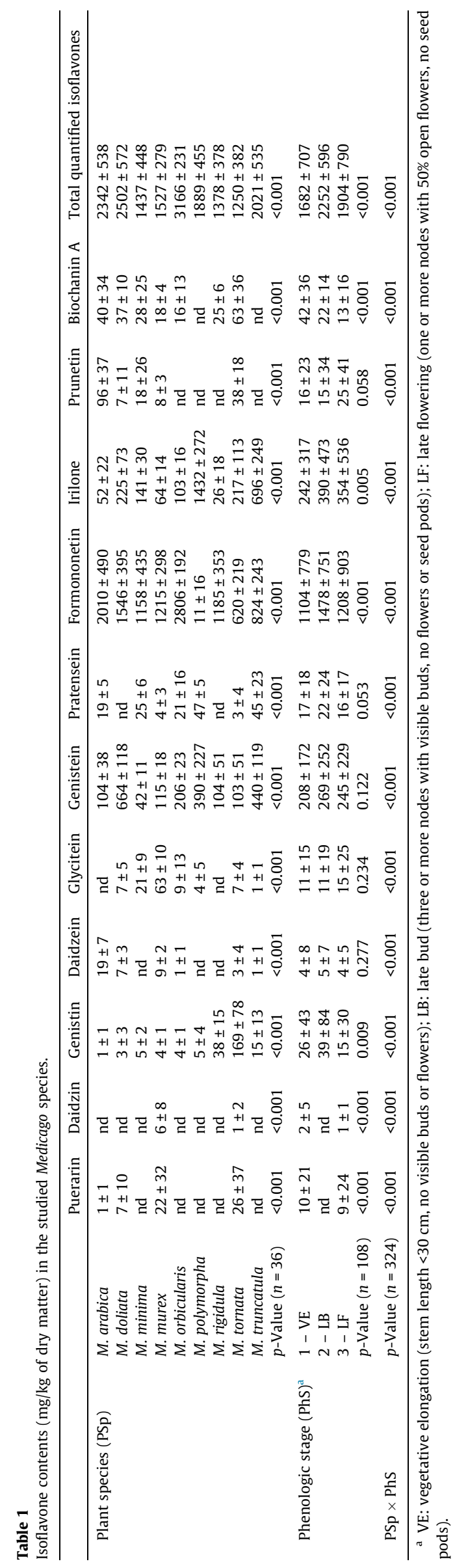


isoflavone composition reported as the mean value of each PSp considering the three $\mathrm{PhS}$, as well as mean value of each $\mathrm{PhS}$, individually containing the values for all nine PSp. Accordingly, the presented standard deviations should not be understood in sensu strictu, since the mean values obtained in each case were calculated using the results given by different PSp or PhS.

As it can be concluded from Table 1, the interaction PSp $\times$ PhS was significant for all isoflavones, meaning that the variation in isoflavones contents resulted from the conjunct action of both factors simultaneously. Accordingly, it was not possible to classify the results through multiple comparison tests (such as Tukey's HSD or Tamhane's T2). Nevertheless, some conclusions could be drawn from the corresponding estimated marginal mean (EMM) plots (data shown only in specific cases).

For instance, late bud (LB) tended to present greater isoflavones levels (except for puerarin and daidzin) independently of PSp. This trend is verified either for individual isoflavones as well as for their total amounts (Fig. 1A-D). The crossed lines are also evidence of the strong interaction between the two factors. These difference are likely to be due to the greater impact of biotic (like pathogen attack) and environmental stresses on plant survival and reproduction in younger plants and the consequent need to maximize plant defense (Bednarek et al., 2001). Actually, the impact of these stress conditions varies within the vegetative cycle, producing different needs in the plant defense system and causing differences in the expression of isoflavones (Posmyk et al., 2005). In comparison with previous results obtained with the same species in the late flower (LF) $\mathrm{PhS}$, a general trend to higher contents in vegetative elongation (VE) and LB was verified for most isoflavones. Besides these quantitative differences, some qualitative dissimilarities were also observed in the obtained profiles. The following isoflavones were not detected in the LF of the indicated species: puerarin in Medicago doliata and Medicago murex; daidzin in M. murex; genistin in Medicago polymorpha; daidzein in Medicago orbicularis and Medicago tornata; glycitein in $M$. doliata, M. polymorpha and $M$. truncatula; pratensein in M. orbicularis; formononetin in M. orbicularis; prunetin in M. doliata; bioachanin A in Medicago arabica, Medicago minima and $M$. orbicularis. The highlighted differences are good indicators of the relevance of the $\mathrm{PhS}$ in which plants should be harvested to benefit from a determined isoflavones' profile. In similar studies (Guo et al., 2011), soybean plants released greater amounts of daidzein and genistein at the pod and seed maturity stages than at the seedling period. A variation in the total isoflavones along the $\mathrm{PhS}$ was also reported in Lupinus albus leaves sowing in different seasons, alfalfa, red clover and soybean (D'Agostina et al., 2008; Seguin et al., 2004). The influence of plant age on the concentration of various isoflavonoids, and specifically the increase in biochanin A and formononetin sugar conjugates,

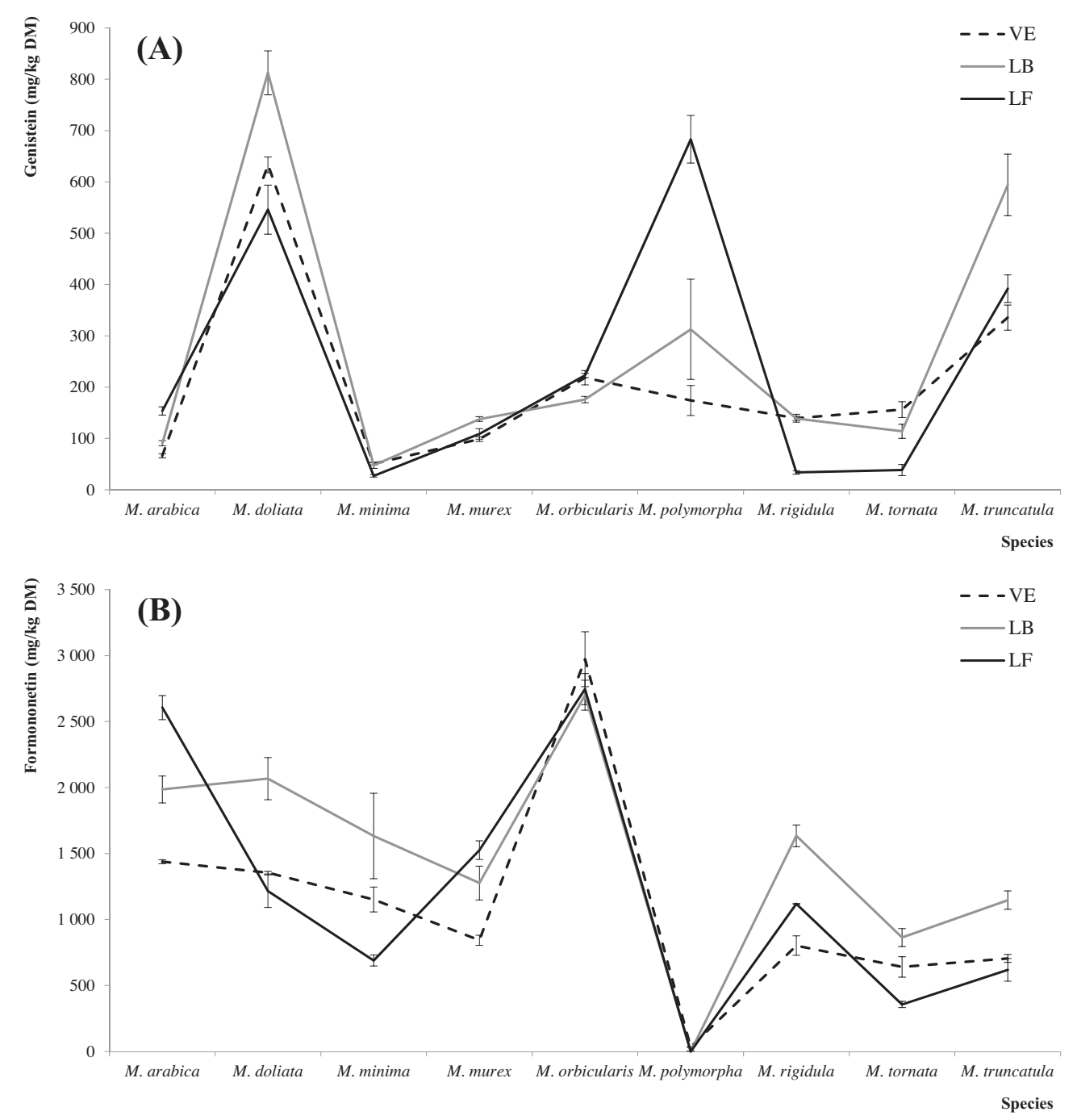

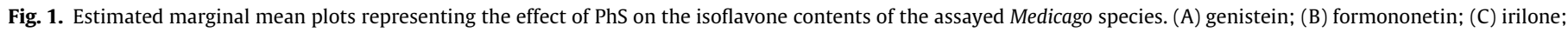
(D) total isoflavones. 

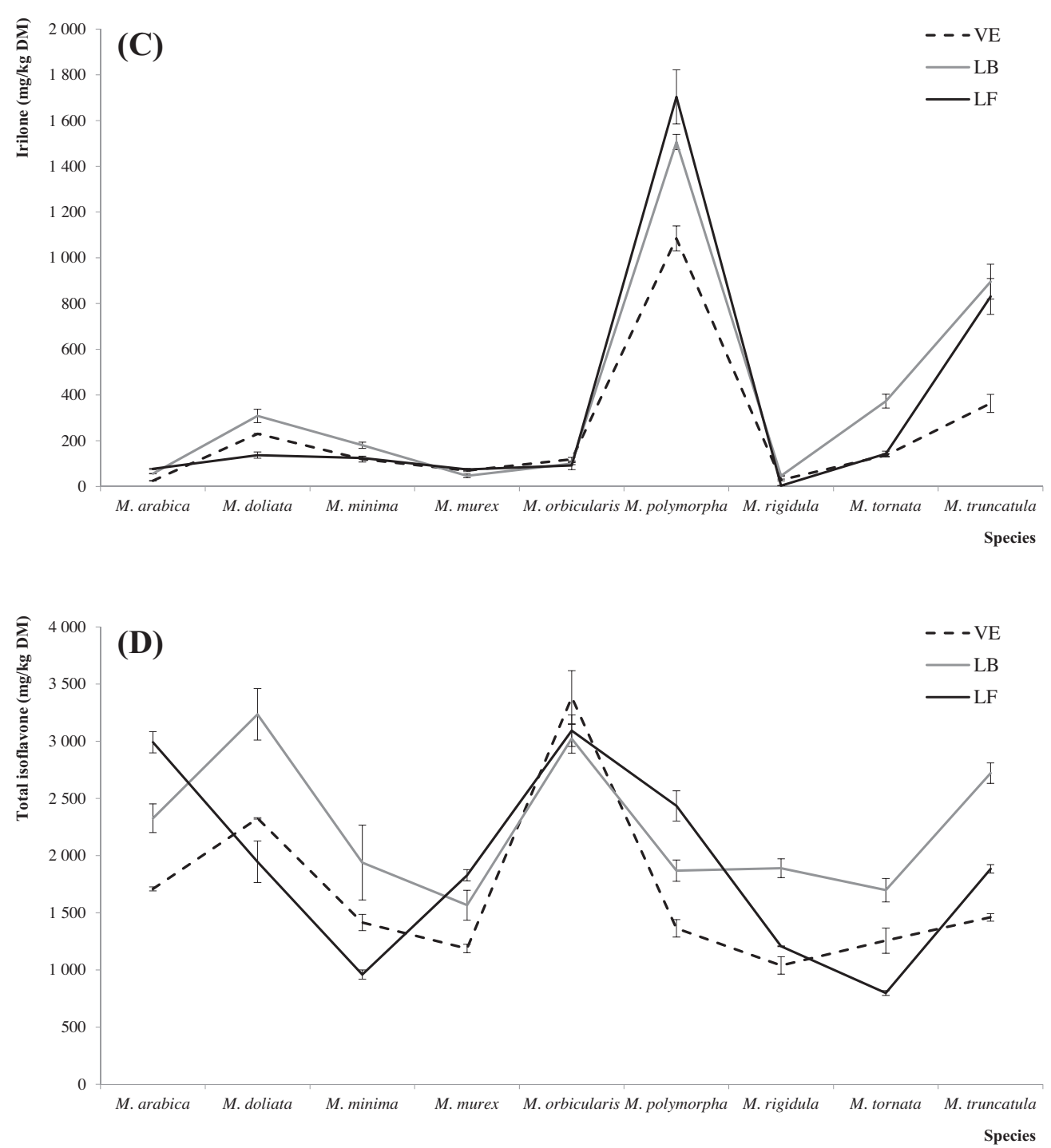

Fig. 1 (continued)

was reported in red clover foliage, where these isoflavone derivatives increased markedly during plant growth (Edwards et al., 1997).

The phylogeny seemed to exert the most marked effects on the production of isoflavones in Medicago leaves. Considering once again the EMM plots (show only for genistein, formononetin and irilone in Fig. 1), and independently of the PhS, genistin and biochanin A tended to be present in higher quantity in $M$. tornata, daidzein and prunetin in $M$. arabica, glycitein in $M$. murex, genistein in $M$. doliata, formononetin in $M$. orbicularis and irilone in M. truncatula. Regarding the isoflavone amounts in a global perspective, $M$. arabica, $M$. doliata and $M$. orbicularis presented the highest overall content of isoflavones (Table 1), while M. tornata presented the lowest.

It was also possible to identify the binomial combination of PSp $\times$ PhS that maximizes the production of each isoflavone: puerarin, $M$. tornata $\times$ late flowering $(\mathrm{LF})$; daidzin, M. murex $\times$ vegetative elongation (VE); genistin, $M$. tornata $\times$ late bud (LB); daidzein, M. arabica $\times \mathrm{VE}$; glycitein, M. murex $\times \mathrm{LF}$; genistein, M. doliata $\times$ LB (detailed in the next paragraph); pratensein, M. truncatula $\times \mathrm{LB}$; formononetin, M. orbicularis $\times \mathrm{VE}$; irilone, M. polymorpha $\times \mathrm{LF}$; prunetin, $M$. arabica $\times \mathrm{LF}$; biochanin $\mathrm{A}$, M. tornata $\times \mathrm{VE}$; total isoflavones, $M$. orbicularis $\times \mathrm{VE}$.
These different combinations are also good indicators of the strong interaction among PhS and PSp. In addition, the evolution of the three major isoflavones (genistein, formononetin and irilone) along the PhS may be observed in Fig. 1A-C. As it can be seen, the variation of these three isofavones along the PhS for each PSp is a striking example of their dissimilar behavior.

Regarding the isoflavone with more reports describing its effects on human health, genistein contents ranged from 27 $($ M. minima $\times \mathrm{LF})$ to $813 \mathrm{mg} / \mathrm{kg} \mathrm{DM}($ M. doliata $\times \mathrm{LB})$, a very high value, even when compared with soybean, in which genistein was reported as the major isoflavone, ranging from $84 \mathrm{mg} / \mathrm{kg}$ to $583 \mathrm{mg} / \mathrm{kg}$ DM along the reproductive stages (Kumar et al., 2009).

In what concerns the total amounts of quantified isoflavones, the obtained values were also higher than those presented by other vegetable species like green bean, carrot, white cabbage, cauliflower, iceberg lettuce or artichoke (Konar et al., 2012). Even regarding soybean, that typically yields $1.4-6.9 \mathrm{~kg}$ (considering $0.6-3.0 \mathrm{~g}$ of isoflavones $/ \mathrm{kg}$ of soybean and 2.3 tons of soybean/ha) of isoflavones (sum of daidzein, genistein, and glycitein in aglycone equivalents)/ha (Mortensen et al., 2009), the productive potential of Medicago spp. might be considered high. These species might reach isoflavone productivities near $50 \mathrm{~kg} / \mathrm{ha}$, considering the average value of total isoflavones in the species assayed herein, and a 
Table 2

Physiological parameters of the evaluated Medicago species grown in the Agrarian Station of Vairão at the experimental field of University of Porto, Portugal.

\begin{tabular}{|c|c|c|c|c|c|c|c|c|c|c|}
\hline \multirow[t]{2}{*}{ Species } & \multicolumn{10}{|c|}{ Phenologic parameters $^{\mathrm{a}}$} \\
\hline & Accession ID & $\mathrm{H}$ & ST & $\mathrm{TE}$ & EpF & $\mathrm{pFF}$ & LC & $\mathrm{E}$ & $\mathrm{pF}$ & $\mathrm{F}$ \\
\hline Medicago arabica & 31,32 & $45 \pm 7 \mathrm{a}$ & $35 \pm 1 c$ & $25 \pm 1 c$ & $118 \pm 14 \mathrm{a}$ & $13 \pm 1 \mathrm{a}$ & $191 \pm 16 \mathrm{ab}$ & $25 \pm 1 b$ & $143 \pm 14$ & $156 \pm 9 a b$ \\
\hline Medicago doliata & 39,41 & $17 \pm 2 b$ & $41 \pm 2 b c$ & $36 \pm 3 b c$ & $124 \pm 2 a$ & $8 \pm 1 b$ & $210 \pm 2 a b$ & $36 \pm 3 \mathrm{ab}$ & $160 \pm 9$ & $168 \pm 7 a$ \\
\hline Medicago minima & 67,68 & $28 \pm 2 \mathrm{ab}$ & $59 \pm 5 b$ & $39 \pm 12 b c$ & $109 \pm 8 \mathrm{ab}$ & $9 \pm 1 \mathrm{ab}$ & $216 \pm 9 \mathrm{a}$ & $39 \pm 5 a b$ & $148 \pm 4$ & $157 \pm 7 \mathrm{ab}$ \\
\hline Medicago murex & 62,69 & $20 \pm 2 b$ & $57 \pm 6$ bc & $48 \pm 5 a b$ & $95 \pm 5 a b$ & $9 \pm 1 \mathrm{ab}$ & $208 \pm 6 a b$ & $48 \pm 5 a$ & $143 \pm 7$ & $152 \pm 4 \mathrm{ab}$ \\
\hline Medicago orbicularis & 66,73 & $42 \pm 7 a$ & $39 \pm 5 \mathrm{bc}$ & $24 \pm 2 c$ & $126 \pm 3 a$ & $10 \pm 1 \mathrm{ab}$ & $199 \pm 1 \mathrm{ab}$ & $24 \pm 4 b$ & $149 \pm 6$ & $160 \pm 6 a b$ \\
\hline Medicago polymorpha & 82,83 & $40 \pm 1 \mathrm{a}$ & $37 \pm 3 \mathrm{bc}$ & $23 \pm 4 c$ & $111 \pm 6 a$ & $11 \pm 1 \mathrm{ab}$ & $182 \pm 5 b$ & $23 \pm 4 b$ & $134 \pm 3$ & $145 \pm 2 b$ \\
\hline Medicago rigidula & 96,97 & $19 \pm 1 b$ & $86 \pm 2 a$ & $18 \pm 2 c$ & $95 \pm 1 \mathrm{ab}$ & $7 \pm 1 b$ & $206 \pm 6 a b$ & $28 \pm 3 b$ & $136 \pm 5$ & $146 \pm 3 a b$ \\
\hline Medicago tornata & 111,116 & $33 \pm 3 a b$ & $40 \pm 10 \mathrm{bc}$ & $28 \pm 5 b c$ & $113 \pm 10 \mathrm{a}$ & $10 \pm 1 \mathrm{ab}$ & $192 \pm 4 \mathrm{ab}$ & $28 \pm 5 b$ & $141 \pm 2$ & $151 \pm 4 a b$ \\
\hline Medicago truncatula & 123,127 & $30 \pm 7 \mathrm{ab}$ & $49 \pm 8$ bc & $66 \pm 4 \mathrm{a}$ & $79 \pm 8 b$ & $8 \pm 1 b$ & $202 \pm 10 \mathrm{ab}$ & $49 \pm 8 a$ & $137 \pm 7$ & $146 \pm 5 a b$ \\
\hline$p$-Value (Tukey's test) & - & 0.001 & $<0.001$ & $<0.001$ & 0.003 & 0.015 & 0.034 & 0.002 & 0.101 & 0.040 \\
\hline
\end{tabular}

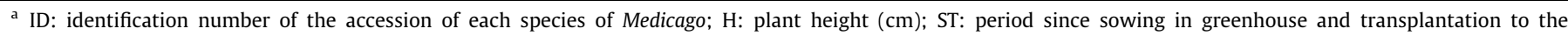

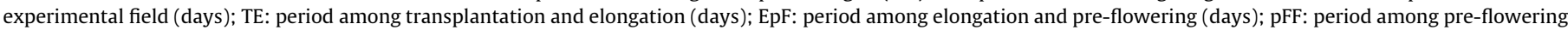

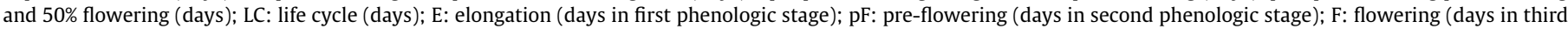
phenologic stage).

productivity of 23 tons of dry matter/ha (Rassini et al., 2000). In addition, all of the most studied isoflavones (genistin, daidzein, glycitein, genistein, formononetin and bioachanin A), thereby with the most well-known activity, were detected in these Medicago species, with special relevance to formononetin. Furthermore, the applied extraction procedure proved to be much more effective than the maceration with polar extracts (Rodrigues et al., 2014).

The differences verified for the phenologic indicators may also explain some of the observed variability. As it can depicted from Table 2, the assayed specimens presented significant differences $(p<0.050)$ for at least one of the assayed species, except for the pre-flowering (days in the second phenologic stage) period $(p=0.101)$. The species with highest isoflavone contents (M. orbicularis, $M$. doliata and M. arabica) presented some common features in their phenology. All their accessions presented low ST (period since sowing in greenhouse and transplantation to the experimental field) and TE (period among transplantation and elongation) and high EpF (period among elongation and pre-flowering) and $\mathrm{F}$ (flowering period). However, it was not possible to completely correlate these differences with the quantified isoflavone amounts. Either way, the qualitative and quantitative differences found among PSp or PhS may result from the variation induced by the type or age of the plants, besides the effect of environmental conditions. In this particular, the stress induced by temperature, light exposure and drought may explain some variation among Medicago species, since plants faced different temperatures during their vegetative development, as it is indicated by the periods of sowing, elongation, transplantation or flowering (Table 2) (Bednarek et al., 2001; Von Baer et al., 2006).

\subsection{Linear discriminant analysis}

The lack of well identified correlations and the high standard deviations resulting from mixing different PSp or PhS hindered obtaining accurate conclusions. Accordingly, it was necessary to apply a statistical classification technique to understand the real variations of isoflavone profiles according to the Medicago species or the phenologic stage in which the plants were obtained. Hence, to obtain a better comprehension about the influence of phylogenetic and phenologic effects over isoflavone profiles, the results were evaluated through LDA, verifying the differentiation power of the selected variables. The significant independent variables (isoflavones) were selected using the stepwise procedure of the LDA, according to the Wilks' $\lambda$ test. Only those with a statistical significant classification performance $(p<0.050)$ were kept for analysis. As it could be expected for the mean values presented in Table 1, the differences in isoflavone profiles gave a good classification performance, allowing classifying correctly $100.0 \%$ of the assayed species for the originally grouped cases as well as for the cross-validated cases. In this discriminant model, six functions were defined as being significant, from which the first three were plotted, (Fig. 2A) integrating $82.4 \%$ of the observed variance (first: $46.0 \%$; second: $23.6 \%$; third: $12.8 \%$ ), with all variables being selected by the model. Function 1, mainly correlated with irilone and formononetin, as deduced from the canonical discriminant functions standardized coefficients (Table 3 ) separated primarily $M$. arabica (high contents in formononetin and low in irilone) and M. polymorpha (low contents in formononetin and high in irilone) from the remaining species. Function 2, more strongly correlated with genistin, projected $M$. tornata (in which genistin was quantified in the highest values) far from any other species. Finally, function 3, more powerfully correlated with glycitein, was particularly effective in separating $M$. murex (supporting the relevance of its high contents in glycitein). Nevertheless, all species were individualized, indicating that the isoflavone profile of each Medicago species is particularly affected by phylogenetic factors.

Regarding the effect of the PhS, the discriminant model defined two significant functions (Fig. 2B) integrating $100.0 \%$ of the observed variance (first: $70.2 \%$; second: $29.8 \%$ ). The classification performance was, as it could also been expected from the $p$-values in Table 1, quite lower than the obtained for the effect of PSp, classifying correctly only $61.7 \%$ ( 18 VE as LB, 42 VE as LF; 5 LB as VE, 29 LB as LF; 22 LF as VE, 8 LF as LB) of the assayed species for the originally grouped cases and $60.2 \%$ ( $18 \mathrm{VE}$ as LB, $42 \mathrm{VE}$ as LF; $5 \mathrm{LB}$ as VE, 29 LB as LF; 22 LF as VE, 13 LF as LB) for the cross-validated cases, with genistein as the only variable being excluded by the model. This result indicates that the $\mathrm{PhS}$, per se, should not be the main concern when electing the optimal conditions for harvesting plants with a desirable isoflavone profile, highlighting that using monoculture crops would be a more reliable option.

Overall, this study evaluated the influence of phenologic and phylogenetic effects on the isoflavone content of Medicago spp leaves grown on open-field conditions, with particular relevance for PSp. Considering the detected isoflavone amounts, Medicago species might be considered as interesting alternative source of these phytoestrogens. The most important criterion to be considered when using these plants as isoflavone sources resulted to be the selected species, since the variation along the vegetative cycle seemed to be less pronounced.

The influence of genotypic factors was also found to be the most relevant source of variation in our previous study with different Fabaceae genera, namely Biserrula, Lotus, Ornithopus and Scorpiurus (Visnevschi-Necrasov et al., 2014b). With the present results, it becomes clear that the genetic modulation of isoflavones' biosynthetic pathway goes beyond the genus, being associated with the lowest taxonomical rank. 

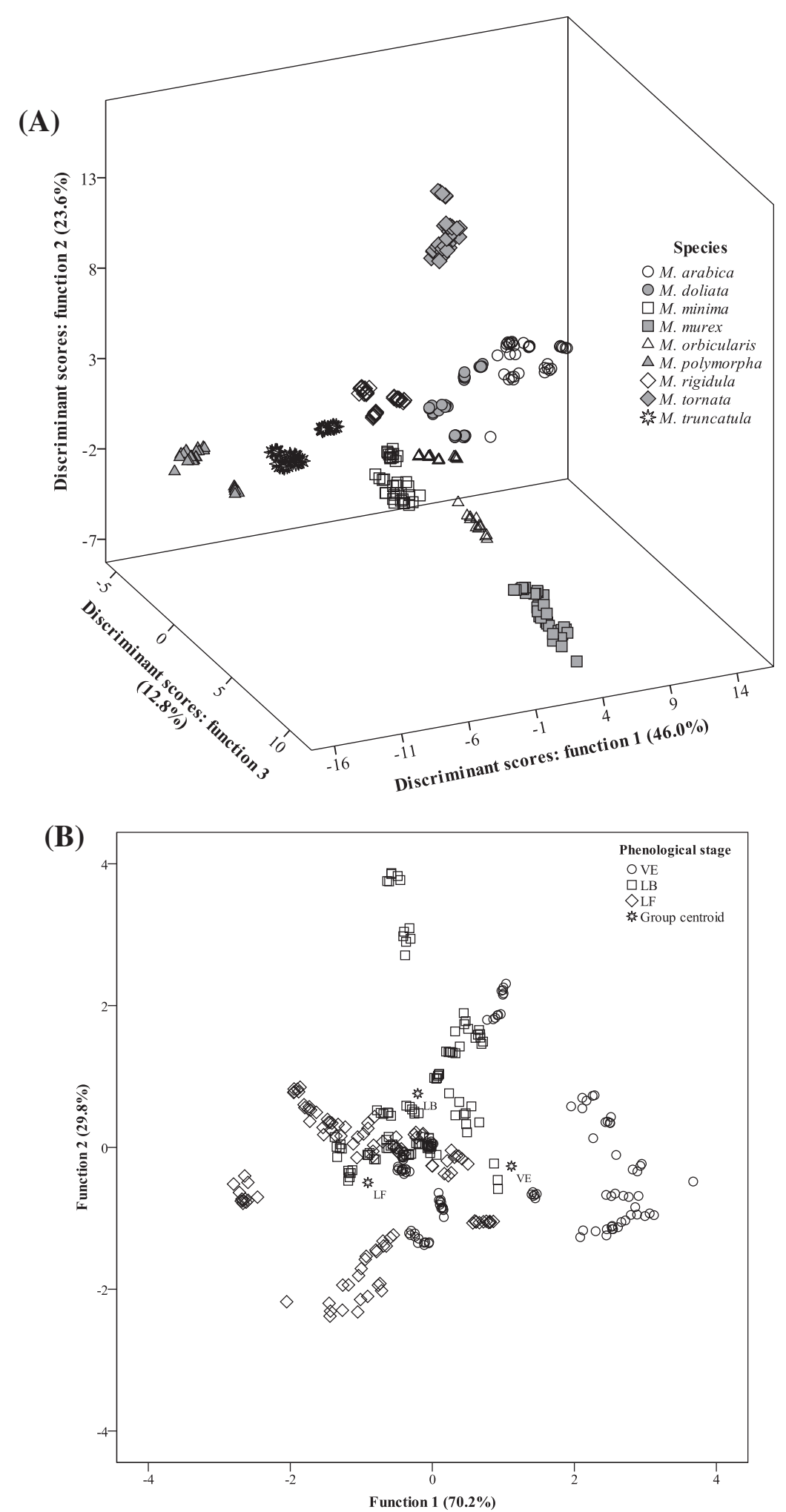

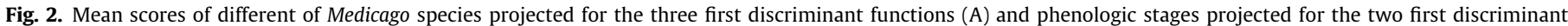
functions (B) defined from the isoflavone profiles.

In addition the best combination of both factors (PSp and PhS) that maximize the yield in a determined isoflavone were found. This provides a more natural way to obtain suitable isoflavone concentrations, in comparison to the metabolic engineering of isoflavonoid biosynthesis. The results obtained in this work might be considered as a step forward in the process of using Medicago spp. as isoflavone sources, particularly because the effects of these compounds are highly dependent on their type and concentration.

Besides their application as foodstuff, leaves of Medicago spp. (especially M. truncatula, $M$. orbicularis and Medicago rigidula) can be useful to the nutraceutical and pharmacological industries as a potential source of isoflavones, particularly genistein and 
Table 3

Pooled within-groups correlations between discriminating variables and standardized canonical discriminant functions.

\begin{tabular}{|c|c|c|c|c|c|c|c|c|}
\hline \multirow[t]{3}{*}{ Isoflavone } & \multicolumn{8}{|c|}{ Discriminant functions } \\
\hline & \multicolumn{6}{|c|}{ PSp effects } & \multicolumn{2}{|c|}{ PhS effects } \\
\hline & 1 & 2 & 3 & 4 & 5 & 6 & 1 & 2 \\
\hline Glycitein & 0.042 & -0.226 & 0.681 & 0.065 & 0.152 & -0.147 & -0.085 & -0.115 \\
\hline Prunetin & 0.166 & 0.123 & -0.089 & 0.364 & -0.029 & -0.266 & -0.110 & -0.176 \\
\hline Genistein $^{\mathrm{a}}$ & -0.056 & -0.077 & -0.249 & -0.209 & 0.618 & 0.498 & -0.126 & 0.108 \\
\hline Formononetin & 0.261 & -0.155 & -0.084 & -0.153 & -0.383 & 0.685 & -0.100 & 0.320 \\
\hline Irilone & -0.392 & -0.042 & -0.261 & 0.341 & 0.428 & 0.644 & -0.139 & 0.142 \\
\hline Pratensein & -0.161 & -0.099 & -0.182 & 0.217 & -0.074 & 0.157 & -0.006 & 0.248 \\
\hline Genistin & -0.016 & 0.368 & 0.143 & -0.051 & -0.032 & 0.091 & 0.062 & 0.299 \\
\hline Biochanin A & 0.083 & 0.149 & 0.043 & -0.010 & 0.0110 & -0.053 & 0.590 & -0.045 \\
\hline Daidzin & 0.013 & -0.029 & 0.156 & 0.029 & 0.066 & -0.021 & 0.264 & -0.240 \\
\hline Daidzein & 0.213 & -0.022 & -0.024 & 0.323 & 0.228 & -0.151 & 0.044 & 0.149 \\
\hline Puerarin & 0.015 & 0.056 & 0.125 & 0.004 & 0.085 & 0.054 & 0.069 & -0.418 \\
\hline
\end{tabular}

${ }^{\text {a }}$ Genistein was excluded by the linear discriminant model when evaluating the PhS effects.

formononetin. These novel applications might boon the increase of agronomical exploitations, either in number, as well as in surface, boosting the local development of economically constrained areas.

\section{Material and methods}

\subsection{General experimental procedures}

Acetonitrile (HPLC grade) and formic acid (analytical grade) were from Merck (Darmstadt, Germany). Purified demineralized water used was from a "Seradest LFM 20" system (Seral, Ransbach-Baumbach, Germany). The eluents were filtered through $0.45 \mu \mathrm{m}$ filters and degassed under reduced pressure and ultrasonic bath. Disposable syringe filter PTFE $0.45 \mu \mathrm{m}$ was from Macherey-Nagel (Düren, Germany). The $\mathrm{C}_{18}$-bonded silica (particle size $55-105 \mu \mathrm{m}$ ) used as sorbent for MSPD was from Waters (Milford, MA, USA).

\subsection{Plant material and field experimental site}

Nine species of Medicago ( $M$. arabica, $M$. doliata, $M$. minima, M. murex, M. orbicularis, M. polymorpha, M. rigidula, $M$. tornata and $M$. truncatula) were sown in November 2008 at the Experimental Field of the University of Porto (Agrarian Station of Vairão). The vegetal germplasm was obtained from the Portuguese collection of Leguminosae provided by the National Institute of Biological Resources (Instituto Nacional dos Recursos Biológicos, I.P.). Voucher specimens of each Medicago species were numbered and deposited in the local herbarium. Samples were collected from February to July in three phenologic stages: (1) vegetative elongation (stem length $<30 \mathrm{~cm}$, no visible buds or flowers); (2) late bud (three or more nodes with visible buds, no flowers or seed pods); and (3) late flower (one or more nodes with $50 \%$ open flowers, no seed pods). For each species and phenologic stage, three independent samples were selected (in different locations within the limits of the indicated Experimental Field) consisting in fresh leaves from randomly selected plants ( 5 plants for each sample) belonging to 2 different accessions; samples were dried at $65^{\circ} \mathrm{C}$ during $72 \mathrm{~h}$ and milled, at particle size of $0.1 \mathrm{~mm}$, using an A11 analysis mill (IKA Werke, Staufen, Germany).

\subsection{Physiological parameters}

The periods required to fulfil each of the considered physiological stages were accurately measured to obtain a more complete characterization of the physiological response of each species to the edaphoclimatic conditions of the experimental site. The assayed parameters (in days) included: (i) period since sowing in greenhouse and transplantation to the experimental field (ST); (ii) period among transplantation and elongation (TE); period among elongation and pre-flowering (EpF); period among preflowering and 50\% flowering (pFF); life cycle (LC); elongation days in first phenologic stage (E); pre-flowering - days in second phenologic stage $(\mathrm{pF})$; flowering - days in third phenologic stage (F).

\subsection{Extraction procedure}

MSPD extraction of isoflavones was performed following a previous method (Visnevschi-Necrasov et al., 2014a). The same amounts of sample, $C_{18}$ and internal standard (2-methoxyflavone) were used. The extraction mixture was transferred to an empty column, connected to a vacuum system, washed with distilled water and the isoflavones were eluted with methanol: $\mathrm{H}_{2} \mathrm{O}(9: 1$, $\mathrm{v} / \mathrm{v}$ ). Before HPLC analysis, the extracts collected in amber vials were filtered through a $0.45 \mu \mathrm{m}$ PTFE membrane. Different samples of two distinct accessions of all species were extracted.

\subsection{HPLC determination of isoflavones}

Purity-corrected individual isoflavones stock solutions $(1 \mathrm{~g} / \mathrm{L})$ were prepared in methanol: $\mathrm{H}_{2} \mathrm{O}(75: 25, \mathrm{v} / \mathrm{v})$. A composite stock standard solution of multiple isoflavones containing $40 \mathrm{mg} / \mathrm{L}$ of each standard: biochanin A ( $\geqslant 97 \%)$, puerarin $(\geqslant 99 \%)$, glycitein $(\geqslant 97 \%)$, daidzein $(\geqslant 98 \%)$, daidzin $(\geqslant 95 \%)$, prunetin $(\geqslant 98 \%)$, genistein $(\geqslant 98 \%)$, genistin $(\geqslant 95 \%)$ and formononetin $(\geqslant 99 \%)$ (Sigma-Aldrich, St. Louis, MO, USA); pratensein and irilone (both $\geqslant 98 \%$ ) (Chromadex Inc., Barcelona, Spain) was prepared. The internal standard (IS) 2-methoxyflavone was obtained from Sigma. A working 2-methoxyflavone solution was prepared in methanol at $1 \mathrm{~g} / \mathrm{L}$. All the solutions were stored at $-20^{\circ} \mathrm{C}$ in amber glass vials when not in use.

Chromatographic analyses were performed following the previously optimized methodology (Visnevschi-Necrasov et al., 2014a). A high-performance liquid chromatograph (Jasco, Tokyo, Japan) equipped with a PU-2080 quaternary pump and a Jasco AS-950 automatic sampler were used. Detection was performed with a multi-wavelength diode-array detector Jasco, MD-2010 and the chromatographic separation of the compounds was achieved under the conditions described previously (Visnevschi-Necrasov et al., 2014a). Data were analyzed using the Borwin-PDA Controller Software (JMBS, Le Fontanil, France). Compounds were identified by chromatographic comparisons with authentic standards and UV spectra. Quantification was made using the calibration curves obtained for each identified isoflavone (DAD at $254 \mathrm{~nm}$ ) based on the internal standard (2-methoxyflavone) method. 
The limits of detection (0.011-0.171 mg/L) and quantification $(0.037-0.569 \mathrm{mg} / \mathrm{L})$, as well as the linearity (correlation coefficients higher than 0.99 in all cases), accuracy (recoveries in the range of $82-104 \%$ ) and repeatability (relative standard deviation values ranged from $4 \%$ to $9 \%$ ) of the method were accepted as previously obtained (Visnevschi-Necrasov et al., 2014a).

\subsection{Statistical analysis}

All extractions were performed in triplicate and each replicate was quantified in duplicate. Data were expressed as means \pm standard deviations. All statistical tests were performed at a 5\% significance level using the SPSS software, version 22.0 (SPSS Inc.).

\subsubsection{General linear model}

An analysis of variance (ANOVA) with type III sums of squares was performed using the GLM (General Linear Model) procedure of the SPSS software. The dependent variables were analyzed using 2-way ANOVA, with the factors "plant species" (PSp) and "phenologic stage" (PhS), fixed to evaluate properly the effects of phylogeny and phenology. With this approach, it was expected to identify the most suitable PhS, independently of the PSp, as well as verifying if a determined PSp would provide optimal isoflavone amounts, regardeless of the PhS. In this analysis, when a statistically significant interaction (PSp $\times \mathrm{PhS}$ ) is detected, the two factors are evaluated simultaneously by the estimated marginal means plots for all levels of each single factor. Alternatively, if no statistical significant interaction is verified, means obtained for each of the assayed factors (PhS or PSp) are compared using multiple comparison tests adequate for homoscedastic (e.g., Tukey's HSD) or heteroscedastic (e.g., Tamhane's T2) distributions.

\subsubsection{Stepwise linear discriminant analysis (LDA)}

In addition, a linear discriminant analysis (LDA) was used to assess the global influence of each factor on isoflavone profile. The basic purpose of these discriminant analyses was estimating the connection between a single categorical dependent variable (Medicago species in the first analysis and phonological stage in the second) and a set of quantitative independent variables (the amounts quantified for each isoflavone). The significant independent variables were selected following the stepwise method of the LDA, according to the Wilks' $\lambda$ test, with the usual probabilities of $F$ (3.84 to enter and 2.71 to remove). Only variables with a statistically significant classification performance $(p<0.05)$ were kept in the analysis. This procedure uses a combination of forward selection and backward elimination procedures, where before selecting a new variable to be included, it is verified whether all variables previously selected remain significant (Palacios-Morillo et al., 2013). With this approach, it is possible to identify the significant variables that contribute in higher extent to the discrimination of a determined set of samples. To verify which canonical discriminant functions were significant, the Wilks' $\lambda$ test was applied. A leaving-one-out cross-validation procedure was carried out to assess the model performance.

\section{Acknowledgments}

Visnevschi-Necrasov and João C.M. Barreira are grateful to "FCT - Fundação para a Ciência e a Tecnologia" for their Grants (SFRH/BD/31734/2006 and SFRH/BPD/72802/2010, respectively). S.C. Cunha is grateful to "POPH-QREN - Tipologia 4.2, Fundo Social Europeu e Fundo Nacional MCTES”.

\section{Appendix A. Supplementary data}

Supplementary data associated with this article can be found, in the online version, at http://dx.doi.org/10.1016/j.phytochem.2015. 04.011 .

\section{References}

Aloui, A., Dumas-Gaudot, E., Daher, Z., Tuinen, D., Aschi-Smit, S., Morandi, D., 2012. Influence of arbuscular mycorrhizal colonisation on cadmium induced Medicago truncatula root isoflavonoid accumulation. Plant Physiol. Biochem. 60, 233-239.

Antunes, P.M., Rajcan, I., Goss, M.J., 2006. Specific flavonoids as interconnecting signals in the tripartite symbiosis formed by arbuscular mycorrhizal fungi, Bradyrhizobium japonicum (Kirchner) Jordan and soybean (Glycine max (L.) Merr.). Soil Biol. Biochem. 38, 533-543.

Bednarek, P., Franski, R., Kerhoas, L., Einhorn, J., Wojtaszek, P., Stobiecki, M., 2001. Profiling changes in metabolism of isoflavonoids and their conjugates in Lupinus albus treated with biotic elicitor. Phytochemistry 56, 77-85.

D’Agostina, A., Boschin, G., Resta, D., Anniccniarico, P., Arnoldi, A., 2008. Changes of Isoflavones during the growth cycle of Lupinus albus. J. Agric. Food Chem. 56, 4450-4456.

Dakora, F.D., Phillips, D.A., 1996. Diverse functions of isoflavonoids in legumes transcend anti-microbial definitions of phytoalexins. Physiol. Mol. Plant P. 49, $1-20$.

Edwards, R., Tiller, S.A., Parry, A.D., 1997. The effect of plant age and nodulation on the isoflavonoid content of red clover (Trifolium pratense). J. Plant Physiol. 150, 603-610.

Farag, M.A., Hufman, D., Lei, Z., Summer, L.W., 2007. Metabolic profiling and systematic identification of flavonoids and isoflavonoids in roots and cell cultures of Medicago truncatula using HPLC-UV-ESI-MS and GC-MS. Phytochemistry 68, 342-354.

Guo, Z.Y., Kong, C.-H., Wang, J.-G., Wang, Y.-F., 2011. Rhizosphere isoflavones (daidzein and genistein) levels and their relation to the microbial community structure of mono-cropped soybean soil in field and controlled conditions. Soil Biol. Biochem. 43, 2257-2264.

Hoeck, J., Fehr, W., Murphy, P., Welke, G., 2000. Influence of genotype and environment on isoflavone contents of soybean. Crop Sci. 40, 48-51.

Huhman, D.V., Sumner, L.W., 2002. Metabolic profiling of saponins in Medicago sativa and Medicago truncatula using HPLC coupled to an electrospray ion-trap mass spectrometer. Phytochemistry 59, 347-360.

Jacobs, A., Wegewitz, U., Sommerfeld, C., Grossklaus, R., Lampen, A., 2009. Efficacy of isoflavones in relieving vasomotor menopausal symptoms - a systematic review. Mol. Nutr. Food Res. 53, 1084-1097.

Konar, N., Poyrazoğlu, E.S., Demir, K., Artik, N., 2012. Effect of different sample preparation methods on isoflavone, lignan, coumestan and flavonoid contents of various vegetables determined by triple quadrupole LC-MS/MS. J. Food Compos. Anal. 26, 26-35.

Kumar, V., Rani, A., Dixit, A.K., Bhatnagar, D., Chauhan, G.S., 2009. Relative changes in tocopherols, isoflavones, total phenolic content, and antioxidative activity in soybean seeds at different reproductive stages. J. Agric. Food Chem. 57, 27052710.

Morrison, M.J., Cober, E.R., Saleem, M.F., McLaughlin, N.B., Frégeau-Reid, J., Ma, B.L., Woodrow, L., 2010. Seasonal changes in temperature and precipitation influence isoflavone concentration in short-season soybean. Field Crop. Res. $117,113-121$

Mortensen, A., Kulling, S.E., Schwartz, H., Rowland, I., Ruefer, C.E., Rimbach, G., Cassidy, A., Magee, P., Millar, J., Hall, W.L., Birkved, F.K., Sorensen, I.K., Sontag, G., 2009. Review: analytical and compositional aspects of isoflavones in food and their biological effects. Mol. Nutr. Food Res. 53, 266-309.

Nunes, C., de Sousa Araújo, S., da Silva, J.M., Fevereiro, M.P.S., da Silva, A.B., 2008. Physiological responses of the legume model Medicago truncatula cv. Jemalong to water deficit. Environ. Exp. Bot. 63, 289-296.

Palacios-Morillo, A., Alcázar, Á., Pablos, F., Jurado, J.M., 2013. Differentiation of tea varieties using UV-Vis spectra and pattern recognition techniques. Spectrochim. Acta A 103, 79-83.

Posmyk, M.M., Bailly, C., Szafranska, K., Janas, K.M., Corbineau, F., 2005. Antioxidant enzymes and isoflavonoids in chilled soybean (Glycine $\max (\mathrm{L}$.$) Merr.) seedlings.$ J. Plant Physiol. 162, 403-412.

Rassini, J.B., Primavesi, A.C., Primavesi, O., Tupy, O., Lêdo, F.J.S., Ferreira, R.P., Botrel, M.A., Alvim, M.J., 2000. Cultivo da alfafa. Embrapa Pecuária Sudeste. Sistemas de Produção (ISSN 1679-1495).

Rodrigues, F., Almeida, I., Sarmento, B., Amaral, M.H., Oliveira, M.B.P.P., 2014. Study of the isoflavone content of different extracts of Medicago spp. as potential active ingredient. Ind. Crop. Prod. 57, 110-115.

Sakamoto, S., Yusakul, G., Pongkitwitoon, B., Paudel, M.K., Tanaka, H., Morimoto, S. 2015. Simultaneous determination of soy isoflavone glycosides, daidzin and genistin by monoclonal antibody-based highly sensitive indirect competitive enzyme-linked immunosorbent assay. Food Chem. 169, 127-133.

Schliemann, W., Ammer, C., Strack, D., 2008. Metabolite profiling of mycorrhizal roots of Medicago truncatula. Phytochemistry 69, 112-146.

Seguin, P., Zheng, W., Souleimanov, A., 2004. Alfalfa phytoestrogen content: impact of plant maturity and herbage components. J. Agron. Crop Sci. 190, 211-217.

Setchell, K.D., Cassidy, A., 1999. Dietary isoflavones: biological effects and relevance to human health. J. Nutr. 129, 758S-767S. 
Silva, L.R., Pereira, M.J., Azevedo, J., Gonçalves, R.F., Valentão, P., de Pinho, P.G., Andrade, P.B., 2013. Glycine $\max$ (L.) Merr., Vigna radiata L. and Medicago sativa L. sprouts: a natural source of bioactive compounds. Food Res. Int. 50, $167-175$.

Stochmal, A., Kowalska, I., Janda, J., Perrone, A., Piacente, S., Oleszek, W., 2009. Gentisic acid conjugates of Medicago truncatula roots. Phytochemistry 70, 1272 1276.

Visnevschi-Necrasov, T., Barreira, J.C.M., Cunha, S.C., Pereira, G., Nunes, E., Oliveira, M.B.P.P., 2014a. Advances in isoflavone profile characterisation using matrix solid-phase dispersion coupled to HPLC/DAD in Medicago species. Phytochem. Anal. http://dx.doi.org/10.1002/pca.2534.
Visnevschi-Necrasov, T., Barreira, J.C.M., Cunha, S.C., Pereira, G., Nunes, E., Oliveira, M.B.P.P., 2014b. Phylogenetic insights on the isoflavone profile variations in Fabaceae spp.: assessment through PCA and LDA. Food Res. Int. http:// dx.doi.org/10.1016/j.foodres.2014.11.032.

Von Baer, D., Saelzer, R., Vega, M., Ibieta, P., Molina, L, Von Baer, E. Ibanez, R. Hashagen, U., 2006. Isoflavones in Lupinus albus and Lupinus angustifolius: quantitative determination by capillary zone electrophoresis, evolution of their concentration during plant development and effect on anthracnose causing fungus Colletotrichum lupini. J. Chil. Chem. Soc. 51, 1025-1029.

Yildiz, F., 2005. Phytoestrogens in Functional Foods. Taylor \& Francis Ltd., Boca Raton. 